\title{
Design, Development and Initial Validation of a Wearable Particulate Matter Monitoring Solution
}

\author{
José G. Teriús-Padrón ${ }^{(凶)}$, Rebeca I. García-Betances, \\ Nikolaos Liappas, María F. Cabrera-Umpiérrez, \\ and María Teresa Arredondo Waldmeyer \\ Universidad Politécnica de Madrid, ETSI Ingenieros de Telecomunicación, \\ 28002 Madrid, Spain \\ \{jterius, rgarcia,nliappas, chiqui,mta\}@lst.tfo.upm.es
}

\begin{abstract}
Air pollution in one of the main problems that big cities have nowadays. Traffic congestion, heaters, industrial activities, among others produce large quantities of Particulate Matter (PM) that have harmful effects on citizens' health. This paper presents the design, development and initial validation of a wearable device for the detection of PM concentration, with communication capacity via WiFi and Bluetooth Low Energy and an end user interface. The results are promising due to the high accuracy of measurements collected by the developed device. This solution is a step forward in empowering citizens to prevent being exposed to high levels of air pollution and is the beginning of what could be a macro-network of air quality sensors within a Smart City.
\end{abstract}

Keywords: Air pollution $\cdot$ Wearable sensor $\cdot$ Smart cities $\cdot$ Particulate matter

\section{Introduction}

Air pollution affects the quality of life (QoL) of people in large cities [1]. Vehicular traffic, heaters and lack of clean transport alternatives are some of the causes of this problem [2]. According to the World Health Organization, only $20 \%$ of cities worldwide comply with the required air quality standards [3].

Among the most dangerous pollutants, the Particulate Matter (PM) is one of the air pollutants that causes most damage to people's health due to its characteristics of size and composition [4]. $\mathrm{PM}_{10}$ or Coarse Particles have a diameter of less than $10 \mu \mathrm{g}$, $\mathrm{PM}_{2.5}$ or Fine Particles less than $2.5 \mu \mathrm{g}$, and $\mathrm{PM}_{1}$ less than $1 \mu \mathrm{g} / \mathrm{m}^{3}$ [5].

Due to the long-term exposure to air pollutants, especially $\mathrm{PM}_{2.5}$, the mortality of people with chronic diseases is increasing [6]. Cardiovascular, respiratory and neurodegenerative diseases are directly affected by the effects of these pollutants $[7,8]$. One of the ways to reduce exposure to pollution is by providing awareness about the pollution levels within the cities. Smart Cities offer data that come from the air quality stations installed in the city from which different services and applications (e.g. PulsAir application [9]) could gather data to empower citizens with urban, environment and 
health recommendations. However, the data currently available come from fixed sensor stations, and do not necessary cover the entire city.

On the other hand, there are few wearable devices available in the market that seek to provide the user with the necessary information about the pollution levels in a precise location of the city. These solutions present certain weaknesses like the Sparrow [10] and ATMO sensors [11] only focus on $\mathrm{C}_{2}$ detection, and The Plume Flow [12] only uses Bluetooth as a communication protocol which limits the possibility to scale-up this solution to other services within a Smart City.

This manuscript presents the design, development and initial validation of a wearable real-time Air Quality (AQ) monitoring device based on PM concentration detection, with a user-friendly interface to show AQ levels, and to report warnings, alerts and recommendations based on the pollution levels detected. The device allows to send the gathered data to other devices or services through WiFi and Bluetooth Low Energy (BLE) and can be programmed to use different communication protocols. This solution is presented as the initial step for a result applicable to large Smart Cities in order to increase the granularity of air quality information that allows the public authorities to develop the appropriate policies to improve the QoL of the citizens.

The next sections are organized as follows: Sect. 2 presents the methodology and material used to develop the proposed solution; Sect. 3 includes the design, develop and evaluation of the system; and finally, Sect. 4 presents discussions, conclusion and future steps to extend this work.

\section{Materials and Methods}

The proposed solution includes the design development and initial validation of the wearable device for air quality measurement, with a user-friendly interface and multiple communication capabilities. The materials used throughout this work are the following:

- PMS7003 Air Quality Sensor (Plantower, Co.): this device can measure concentrations of $\mathrm{PM}_{1}, \mathrm{PM}_{2.5}$, and $\mathrm{PM}_{10}$. It has a built-in flow system that allows air circulation through the device enabling to measure pollution levels.

- ESP32 microcontroller (Espressif Systems, Co.): it can establish communication via Serial, WiFi and BLE protocols, which gives great versatility when transmitting or receiving data to/from sensors, actuators and services.

- Sketch-Up software (Trimble, Inc.): is the software used to design the 3D case of the wearable device to be printed in the Ultimaker 3 extended (Ultimaker BV).

- Air Visual Pro Air Quality device (IQAir, Inc): a commercial fixed air quality device, capable to measure $\mathrm{PM}_{1}, \mathrm{PM}_{2.5}$, and $\mathrm{PM}_{10}$. The device has a test configuration, that allows to run tests and extract the data collected.

- Arduino Integrated Development Environment (IDE): is an open-source development software to program multi-platform microcontrollers boards [13]. 
The following methodology has been established:

\section{First Phase, state of the art study of $A Q$ sensors and protocols:}

In this phase, a research of the main air pollutants and their impact on the people health was carried out. Additionally, air quality protocols were studied in order to select the one to be applied in our system, based on current regulations and standards. Finally, a study of air quality sensors available in the market and comparisons were done in terms of size, accuracy communication and energy consumption. Findings of this phase were used to design the proposed device.

\section{Second Phase, design and development of the solution:}

Once the AQ sensor was selected the design and development of the electronics necessary for the operation of the device were conducted. As a final stage, the wearable case, where all the technology developed is built in, was designed with the $3 \mathrm{D}$ design sketch tool and produced in the 3D printer.

\section{Third Phase, initial validations:}

This phase consisted on analysing the operation of the device according to the established design, making the necessary adjustments and comparisons with a commercial device to study its accuracy.

Tests were carried out following two stages to analyse the measured quality of the solution developed compared with the Air Visual Pro air quality monitor. In the first stage, the PM sensor was placed without any encapsulation. In the second stage, the same test was conducted with the final design (including the case) in order to verify that the design of the 3D encapsulation did not affect the sensor accuracy. The devices were placed in a home kitchen and synchronized to measure $\mathrm{PM}_{2.5}, \mathrm{PM}_{1}$ and $\mathrm{PM}_{10}$, every five minutes. These tests were carryout for a total of six days, three continuous days in each test.

\section{Results}

This section presents the results obtained from the design, development and initial validations according to the methodology described in Sect. 2.

\subsection{Main Components and Design}

The designed solution has been conceived with the purpose of being wearable, easy to use and with multiple communication capacity. There are 5 main components: (1) a microcontroller, that mange the sensor, actuators and interface, makes the necessary calculations, and establishes the communications via BLE or WiFi to other devices. It has a lithium battery port and an embedded charger module, which guarantees the portability of the solution; (2) a sensor, that detects the $\mathrm{PM}_{2.5}, \mathrm{PM}_{1}$ and $\mathrm{PM}_{10}$ concentration and sends it to the microcontroller via serial communication; (3) a screen to show the levels of air quality detected by the sensor; (4) a button which has the functionality to activate the screen. In this way, battery consumption is decreased by 
not activating the screen unless the user decides to do so; and (5) a sound emitter, which is activated in case of any health risk due to high pollution levels.

\subsection{Development}

For the development of the firmware, the Arduino IDE has been used taking advantage of the compatibility with the ESP32 microcontroller.

The Air Quality Index (AQI) has been calculated with the data received through the sensor, based on the Environmental Protection Agency standard [14], where 6 levels of pollution are established according to the concentration level of PM. The system will respond in different ways, depending on the AQI value, as presented in Fig. 1.

Three functionalities have been established: to sense the pollution levels, to show the data collected, and to present the information through a user-friendly interface. The measurements are later sent to other devices or services. The functionalities established are the following:

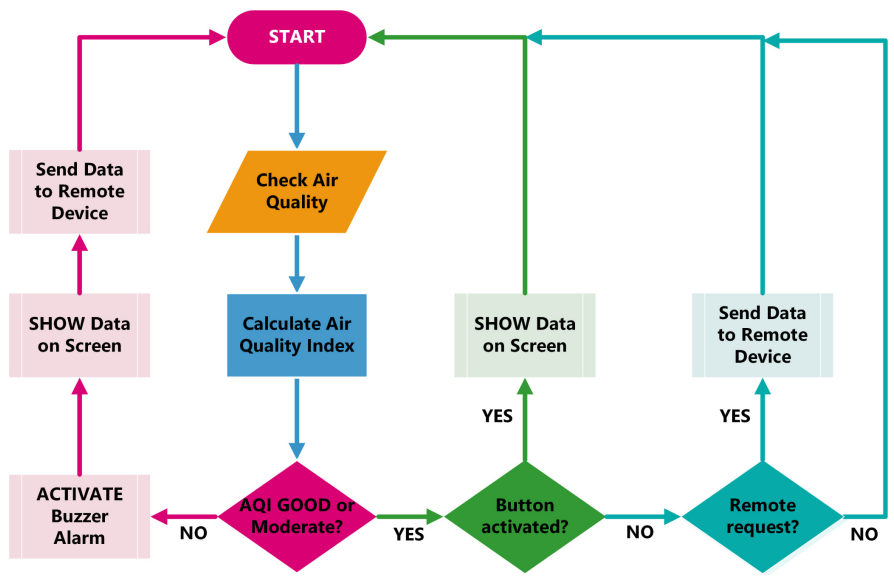

Fig. 1. Flow diagram of the solution.

1. Activation by button: when the button is pressed the device calculates the AQI and shows on the screen the AQI levels, taking into consideration the colour scale described by the AQI standard.

2. Remote communication: the device measurements and the calculated PM concentration are sent, via BLE, to another device (e.g. smartphone) or service. This functionality allows to present the historical data in a mobile application or to be used by other services.

3. Pollution levels: the device reacts autonomously depending on the AQI calculated. When the levels are in a safe range for health $\left(\mathrm{PM}_{2.5}\right.$ concentration between 0 and 
$12 \mu \mathrm{g} / \mathrm{m}^{3}$ ), the device remains in passive mode. However, when the detected levels are harmful $\left(\mathrm{PM}_{2.5}\right.$ concentration above $\left.35.5 \mu \mathrm{g} / \mathrm{m}^{3}\right)$, the system proceeds to alert the user by emitting an alarm sound and showing on the screen the appropriate icon. Furthermore, if a device or service are connected, the information is sent in order to have a historical record of the event.

Each of the functionalities described above were developed individually, and later assembled and incorporated into the case.

\section{3D Case Design}

Taking into account that the users of this device can have different needs, the case has one top cover, were all the components developed are embedded, and then includes three exchangeable lower caps, describes as follows: (1) lower cap with a clip to attach the device on the side of the pants or belt; (2) lower cap with a hole in one side to hang it on bags or purses; (3) lower cap with two holes to be used with an arm band, designed for people who do physical activities.

\subsection{Initial Validations}

As described in Sect. 2, the validation was carried out in 2 phases in order to ensure that the design of the 3D encapsulation did not affect the sensor accuracy. The first, with the prototype developed not embedded in the designed case, and the second, with the final prototype inside the case. In both phases was calculate the mean of the PM concentration the accuracy and the correlation between the designed solution and the Air Visual Pro monitor.

As a result of the first phase, the $\mathrm{PM}_{2.5}$ mean obtained had an accuracy of $91.42 \%$ and a positive correlation of 0.86 , which shows that the PMS7003 sensor has an excellent performance for the detection of $\mathrm{PM}_{2.5}$. Regarding measurements of $\mathrm{PM}_{1}$ and $\mathrm{PM}_{10}$, a similar correlation can be observed however, the accuracy decreases but remains over $75 \%$.

On the second tests, the measurement of $\mathrm{PM}_{2.5}$ concentration has an accuracy of 92.69\% and a correlation of 0.87 . In the case of $\mathrm{PM}_{1}$ and $\mathrm{PM}_{10}$ measurements, the data collected show a high accuracy, over $70 \%$, and a positive correlation.

\section{Discussions and Conclusion}

The system developed represents a novel monitoring device that will help improving the quality of life of people within a smart city environment. Thanks to its small design and wearability, users can have air quality information when moving around city areas where the monitoring stations do not cover. Big cities have insufficient air quality 
monitoring stations, hence there is no granularity in the provided air pollution data, so that the impact that public policies have on pollution levels cannot be measured with total accuracy.

The proposed solution can be useful to implement a macro network of distributed air quality sensors, monitoring the pollution levels in every corner of the city and thus implement more efficient and effective public policies that will impact positively in the quality of life of the citizens. Taking advantage of the portability and communication capabilities of both BLE and WiFi technologies, the developed sensor can be easily scalable and adaptable to other communication systems and protocols such as Web of Things [15]. The initial validation demonstrates that there are no differences in the measures made by the proposed solution and the commercial device Air Visual Pro monitor.

Future research lines include: (1) the conduction of usability tests in order to improve the design of the final product; (2) largest validations of the device with respect AQ monitoring stations in major cities; and (3) the incorporation of this technology as part of an innovative distributed sensor network for large Smart Cities.

Acknowledgment. This work has been funded by the European Union Horizon 2020 research and innovation program under the Marie Skłodowska- Curie grant agreement ACROSSING No 676157.

\section{References}

1. Kelly, F., Fussell, J.: Air pollution and public health: emerging hazards and improved understanding of risk. Environ. Geochem. Health 37, 631-649 (2015)

2. Ambient air pollution: Pollutants. www.who.int/airpollution/ambient/pollutants/en

3. Health, environment and sustainable development: Air Pollution. https://www.who.int/ sustainable-development/cities/health-risks/air-pollution/en/

4. Kim, K., Kabir, E., Kabir, S.: A review on the human health impact of airborne particulate matter. Environ. Int. 74, 136-143 (2015)

5. Zhang, L., Ninomiya, Y., Yamashita, T.: Formation of submicron particulate matter (PM1) during coal combustion and influence of reaction temperature. Fuel 85, 1446-1457 (2006)

6. Cohen, A., Brauer, M., Burnett, R., Anderson, H., Frostad, J., et al.: Estimates and 25-year trends of the global burden of disease attributable to ambient air pollution: an analysis of data from the global burden of diseases study 2015. Lancet 389, 1907-1918 (2017)

7. Zaheer, J., Jeon, J., Lee, S., Kim, J.: Effect of particulate matter on human health, prevention, and imaging using PET or SPECT. Prog. Med. Phys. 29, 81 (2018)

8. Underwood, E.: The polluted brain. Science 355, 342-345 (2017)

9. Ottaviano, M., Beltrán-Jaunsarás, M., Teriús-Padrón, J., et al.: Empowering citizens through perceptual sensing of urban environmental and health data following a participative citizen science approach. Sensors 19(13), 2940 (2019)

10. Sparrow Sense. https://www.sparrowsense.com/

11. AMTO. https://atmotube.com/?view=es

12. Plume Labs. https://plumelabs.com/en/flow/

13. Boxall, J.: Arduino Workshop: A Hands-on Introduction with 65 Projects. No Starch Press (2013) 
14. US Environmental Protection Agency, US Environmental Protection Agency, Office of Air Quality Planning and Standards, Outreach and Information Division. Air Quality Index: A guide to air quality and your health. EPA-456/F-14-002 (2014)

15. Teriús-Padrón, J., et al.: Autonomous air quality management system based on Web of Things standard architecture. In: The 5th IEEE Smart World Congress (In press)

Open Access This chapter is licensed under the terms of the Creative Commons Attribution 4.0 International License (http://creativecommons.org/licenses/by/4.0/), which permits use, sharing, adaptation, distribution and reproduction in any medium or format, as long as you give appropriate credit to the original author(s) and the source, provide a link to the Creative Commons license and indicate if changes were made.

The images or other third party material in this chapter are included in the chapter's Creative Commons license, unless indicated otherwise in a credit line to the material. If material is not included in the chapter's Creative Commons license and your intended use is not permitted by statutory regulation or exceeds the permitted use, you will need to obtain permission directly from the copyright holder. 